\title{
Philosophiques
}

\section{De quoi le zoocentrisme est-il le symptôme ?}

Étude critique de l'ouvrage d'Étienne Bimbenet, Le complexe des trois singes. Essai sur l'animalité humaine

\section{Pierre-Jean Renaudie}

Volume 47, numéro 2, automne 2020

URI : https://id.erudit.org/iderudit/1075137ar

DOI : https://doi.org/10.7202/1075137ar

Aller au sommaire du numéro

Éditeur(s)

Société de philosophie du Québec

ISSN

0316-2923 (imprimé)

1492-1391 (numérique)

Découvrir la revue

Citer cet article

Renaudie, P.-J. (2020). De quoi le zoocentrisme est-il le symptôme ? Étude critique de l'ouvrage d'Étienne Bimbenet, Le complexe des trois singes. Essai sur l'animalité humaine. Philosophiques, 47(2), 493-502.

https://doi.org/10.7202/1075137ar d'utilisation que vous pouvez consulter en ligne. 


\title{
Étude critique
}

\section{De quoi le zoocentrisme est-il le symptôme?}

\author{
Étude critique de l'ouvrage d'Étienne Bimbenet, Le complexe des trois \\ singes. Essai sur l'animalité humaine
}

\author{
PIERRE-JEAN RENAUDIE \\ Université Lyon 3
}

Nous sommes tous darwiniens: que nous le voulions ou non, les travaux de Darwin sur l'évolution ont reconfiguré le cadre à l'intérieur duquel nous pouvons comprendre la place de l'homme dans le monde, œuvrant à substituer à une pensée métaphysique de la spécificité humaine une forme de continuisme qui nous a appris à repenser la différence entre l'homme et l'animal comme une différence de degré plutôt que de nature. Ce constat constitue le point de départ du dernier ouvrage d'Étienne Bimbenet (Le complexe des trois singes, Paris, Seuil, 2017): un peu moins de cent cinquante ans après la publication de The Descent of Man (La filiation de l'homme et la sélection liée au sexe, publié par Darwin en 1871), c'est maintenant une cause entendue, nous sommes essentiellement des animaux, et seulement secondairement humains. Nous avons appris à reléguer notre humanité au second plan en la traitant comme un caractère seulement secondaire de l'espèce animale à laquelle nous prétendons désormais appartenir. Les thèses évolutionnistes de Darwin ont exercé une influence si profonde sur les cadres de pensée de l'anthropologie qu'elles ont contribué à définir la «nouvelle figure de notre humanité » qui est aujourd'hui la nôtre, enregistrant à ce titre une avancée (et dans une certaine mesure un progrès) sur lequel il ne peut plus être question de revenir. Nous sommes passés aujourd'hui d'un anthropocentrisme à un zoocentrisme, qui relativise la spécificité de l'humain en n'y voyant qu'une forme d'animalité particulière, et ce faisant nous avons changé de référent pour comprendre notre propre finitude: celle-ci ne nous est plus indiquée par l'infinité de Dieu à laquelle elle s'oppose, mais par notre proximité avec l'animal auquel l'homme s'identifie aujourd'hui pleinement.

Ce constat de Bimbenet appelle d'abord un diagnostic précis, dont l'objectif consiste moins à remettre en cause le contenu de la thèse qui soustend ce zoocentrisme, qu'à en interroger la signification sur un mode permettant d'en déplier les enjeux théoriques. Le problème, en d'autres termes, est moins ce que le discours zoocentrisme affirme que ce qu'il cache et retire à la prise de tout jugement critique. D'un point de vue philosophique, l'évidence dont se pare le zoocentrisme est d'autant plus suspecte qu'elle est moins sujette à caution, se posant comme un acquis du progrès scientifique et culturel des 150 dernières années qu'il ne saurait plus être question de 
soumettre à la discussion. Mais en devenant le présupposé obligé, inquestionnable et inquestionné, de notre compréhension de nous-mêmes, le zoocentrisme nous retire notre capacité à réfléchir sur nous-mêmes, et à ce titre il fournit les coordonnées d'une nouvelle forme d'obscurantisme constituant, sous couvert de scientificité et d'une morale qu'il ne peut de toute façon plus être aujourd'hui question de refuser, un obstacle qui frappe d'interdiction plus qu'il ne libère la pensée des rapports entre l'homme et l'animal. Aussi le changement de paradigme que le zoocentrisme accomplit repose-t-il, aux yeux de l'auteur, sur un coup de force théorique excluant $a$ priori toute forme de réflexion critique sur ses enjeux philosophiques.

C'est à l'analyse de ce coup de force qu'est consacré le Complexe des trois singes, qui nous invite à reconnaître dans le changement de paradigme auquel le zoocentrisme a donné lieu une mutation de la doxa reconduisant inévitablement le dogmatisme propre à la métaphysique qu'il prétend paradoxalement dépasser. Élargissant le cadre de la réflexion philosophique qu'il a consacrée à la question de la différence anthropologique depuis la publication en 2004 de son ouvrage sur l'œuvre de Merleau-Ponty ${ }^{1}$, Étienne Bimbenet s'attache dans ce nouvel ouvrage à mettre en évidence les enjeux contemporains de ce questionnement et à répondre aux défis que le zoocentrisme contemporain lui impose. Encore une fois, entendons-nous bien: nul ne peut revenir aujourd'hui sur les acquis de la révolution darwinienne, et Bimbenet ne propose pas davantage que les «animalistes " de revenir à une conception métaphysique de la spécificité humaine. Loin de rejeter les acquis incontestables de la révolution darwinienne, son analyse doit nous amener à reconnaître que, si la thèse de l'animalité humaine n'est pas en soi fausse, elle ne nous laisse apercevoir qu'une partie tronquée et incomplète de nous-mêmes, et fait resurgir une forme perverse de métaphysique en maintenant le cadre obsolète du questionnement qu'elle prétendait dépasser.

Il s'agit donc de mettre à l'épreuve la cohérence interne du zoocentrisme plutôt que d'en rejeter les thèses de l'extérieur, ce qui suppose de déceler ce qui reste de métaphysique dans la position continuiste, dont les arguments font systématiquement resurgir en sous-main des "propres de l'homme» dont elle nous avait faussement promis de nous débarrasser. S'autorisant d'une science fondée sur les avancées de la biologie de l'évolution, des neurosciences cognitives et de l'éthologie animale, mais dont la valeur philosophique des résultats n'est jamais clairement établie, s'appuyant sur une moralisation de la question animale qui refuse a priori de laisser la réflexion philosophique s'engager sur une voie pouvant conduire à établir des distinctions qu'elle perçoit uniquement comme des formes de discrimination potentielles de l'animal, se réclamant enfin d'une critique de

1. Nature et humanité, le problème anthropologique dans l'œuvre de Merleau-Ponty, Paris, Vrin, 2004; L'animal que je ne suis plus, Paris, Gallimard, 2011; L'invention du réalisme, Paris, Cerf, 2015. 
la métaphysique qui définit peu ou prou les coordonnées contemporaines du «philosophiquement correct», le zoocentrisme refuse une approche fine et nuancée du jeu de similitudes et de différences entre l'homme et les animaux qui permettrait de donner un sens philosophique à leur relation, au lieu d'en présupposer la nature en les identifiant de force les uns aux autres. S'il rappelle en cela le motif des trois singes de la sagesse confucéenne - qui refusent de voir, s'interdisent d'entendre, et s'abstiennent de dire - le complexe intellectuel que le zoocentrisme recouvre a un coût, et un coût assez exorbitant pour l'homme, que Bimbenet diagnostique comme une forme nouvelle d'aliénation. Parce qu'il place à l'extérieur de l'homme les conditions de son auto-compréhension, le zoocentrisme le dépossède de son savoir sur soi: nous ne pouvons plus dire qui nous sommes. L'ère du zoocentrisme marque ainsi l'apogée d'une forme de pensée au moyen de laquelle l'homme contemple sa propre vie de l'extérieur, comme s'il en avait perdu les clés et avait renoncé à en être l'acteur, préférant à une connaissance fondée sur l'expérience en première personne que chacun peut faire de sa propre humanité un savoir de soi fondamentalement «désengagé ».

Il était donc grand temps de reprendre l'enquête en faisant de la lucidité, ainsi que le demande Bimbenet, la vertu cardinale d'une réflexion sur la «différence animale» faisant droit à la spécificité de l'humain. "Guide des égarés à l'âge du zoocentrisme", tel aurait pu être le titre donné par Étienne Bimbenet à ce dernier ouvrage, dans la mesure où il se donne à la fois pour tâche de permettre au lecteur de se repérer dans l'intense maquis théorique des formes complexes et sophistiquées de naturalisme sur lequel le zoocentrisme fait fond, et de lui donner les moyens de s'y orienter en s'appuyant sur les éléments de réflexion proposés par Bimbenet dans la seconde partie de l'ouvrage ("pour un anthropocentrisme élargi»). Encore est-il nécessaire, afin d'apporter des éléments de réponse aux apories sur lesquelles débouche le rejet contemporain de l'anthropocentrisme, de commencer par en produire une analyse systématique et critique, laquelle constitue l'objet de la première partie de l'ouvrage ( «un nouveau fétiche»).

Le zoocentrisme doit, selon Bimbenet, sa force aux trois puissantes motivations - scientifique, morale, philosophique - qui le portent et le soutiennent, et en lesquelles on peut diagnostiquer trois formes différentes d' "oubli» de l'humain que l'auteur analyse en détail dans cette première partie. Ces trois motivations œuvrant de façon plus ou moins explicite à nous rendre insensibles à la spécificité de l'humain sont constitutives du fameux «complexe des trois singes » caractéristique du paradigme zoocentriste, dont le déploiement et le succès sont d'autant mieux garantis selon l'auteur que l'on s'est d'abord rendus sourds à l'enseignement dont sont porteuses les sciences humaines ("ne rien entendre»), que l'on a accepté de taire ce dont la prise en considération serait susceptible de contrevenir aux principes intangibles de la nouvelle morale animaliste («ne rien dire»), et que l'on s'est pour finir rendus avengles aux traits essentiels de l'humain 
qu'une analyse philosophique débordant le cadre du naturalisme pourrait nous permettre d'avoir en vue («ne rien voir»).

\section{La légitimation scientifique}

Le zoocentrisme obéit d'abord à une motivation d'ordre scientifique à laquelle il doit incontestablement son succès et qui lui offre ses gages de crédibilité: en généralisant le traitement de la différence anthropologique comme une simple différence de degré, le zoocentrisme reprend à son compte le programme d'un naturalisme fondé sur la biologie évolutionniste auquel il procure une forme d'accomplissement (chapitre II). Il épouse ainsi parfaitement le processus de "renaturalisation» de l'édifice du savoir que nous sommes en train de vivre aujourd'hui, lequel se fonde essentiellement sur les succès et les avancées de la biologie. Or ce mouvement s'accompagne inévitablement d'une secondarisation du discours sur l'homme dont les sciences humaines sont porteuses, et qui ne peut avoir de valeur que dérivée dès lors que la biologie évolutionniste, l'éthologie, les neurosciences, ou les sciences cognitives peuvent revendiquer une prise en charge des questions qui étaient jusqu'alors celles de la psychologie, de la sociologie ou de la philosophie de l'esprit. L'évolutionnisme, le cognitivisme et l'éthologie animale constituent ainsi les trois piliers d'un programme scientifique naturaliste visant à rattacher les sciences humaines à la biologie en redéfinissant l'humanité à l'aune de notre origine animale et en s'efforçant de démontrer l'universalité des processus cognitifs à l'œuvre dans le vivant. Mais ce qui se joue dans l'arrièreboutique de ce naturalisme dont l'abolition des frontières entre l'humain et l'animal constitue le fonds de commerce, c'est paradoxalement, comme le montre de façon très convaincante Étienne Bimbenet, le retour en force de l'idée de nature humaine, ne se présentant certes plus comme l'étendard d'une conception métaphysique dépassée de l'homme, mais comme fondée dans les sciences du vivant, à qui l'on confie désormais le soin d'établir des caractères propres à l'espèce humaine. C'est donc moins au dépassement des apories auxquelles aboutissait la conception métaphysique de l'humain que l'on assiste, qu'à un transfert de pouvoir à l'issue duquel on a retiré à la métaphysique - pour mieux les confier à la biologie - le droit et la tâche de nous apprendre ce qui constitue le propre de l'homme. Dans un cas comme dans l'autre, on continue à raisonner dans des termes strictement similaires, que le zoocentrisme échoue manifestement à renouveler.

La thèse de l'animalité humaine s'appuie ainsi moins sur les résultats des sciences du vivant que sur la doxa dans l'ombre de laquelle s'élaborent ces dernières, raison pour laquelle elle est - contrairement aux propositions de la science - incontestable en principe, preuve s'il en est qu'elle n'est pas du même ordre que ces dernières. Qu'elle soit vraie ou fausse, cette thèse relève ainsi essentiellement d'un parti pris faisant partie intégrante, comme l'écrivait déjà Francis Wolff, dont Bimbenet reprend l'argument directeur, de «l'armature conceptuelle des sciences de la vie » : l'animalité humaine exprime 
le présupposé méthodologique plutôt que le point d'aboutissement des sciences du vivant ${ }^{2}$. L'analyse critique de ce premier aspect du zoocentrisme appelle ainsi à la prise en considération d'une approche différente et originale du phénomène humain que celui-ci occulte, en concevant l'apport des sciences humaines en la matière comme un type de connaissance de l'humain complémentaire plutôt que concurrent de la biologie.

\section{La légitimation morale}

La deuxième motivation identifiée par Bimbenet comme constituant l'un des piliers d'angle du zoocentrisme contemporain s'établit sur le terrain de la morale et s'exprime dans la moralisation de nos rapports avec les animaux engagée depuis une cinquantaine d'années. Or, si ce processus de moralisation répond à une transformation de notre sensibilité à l'égard de la souffrance animale récemment mise en évidence par Tristan $\mathrm{Garcia}^{3}$, Bimbenet montre de façon très convaincante qu'il traduit aussi, et peut-être surtout, une redéfinition de notre rapport à nous-mêmes et du sens que nous voulons donner à notre humanité. Ce parti pris relatif à la souffrance animale, la requalification en termes essentiellement moraux des rapports qui nous lient aux «animaux non humains » et la mise en majesté du caractère intrinsèquement éthique de la question animale vont de pair avec une redéfinition des limites à l'intérieur desquelles nous concevons notre humanité, lesquelles imposent un cadre moral à notre réflexion sur nous-mêmes. La question que soulève le troisième chapitre de l'ouvrage consiste à se demander de quel type de raisons procède notre désir d'égaliser les conditions entre les animaux et les hommes en leur ouvrant le domaine du droit et de la considération morale. Que ce mouvement d'égalisation se fonde sur notre sensibilité à la souffrance animale permet au mieux d'en identifier une cause (si tant est que celle-ci n'en soit pas aussi un effet), mais ne nous dégage nullement de la tâche consistant à en identifier les raisons.

En s'interrogeant sur les modalités de la requalification de notre rapport à nous-mêmes que la moralisation de nos rapports aux animaux engage, Bimbenet propose une analyse des raisons qui sous-tendent ce mouvement, dont il montre comment il opère une redéfinition du droit. En quoi en effet le nouveau moralisme des éthiques animales vient-il redéfinir les limites du cadre à l'intérieur duquel nous pouvons concevoir notre humanité ? En ceci précisément qu'il nous interdit de projeter sur notre humanité davantage que ce que nous partageons avec les animaux. Et ici, c'est parce que nous confions à la notion de vie plutôt qu'à celle d'humanité le soin de définir ce qui nous est le plus propre que nous pouvons élargir notre morale à l'ensemble des êtres vivants et étendre le domaine du droit aux êtres qui parti-

2. Francis Wolff, Notre humanité. D’Aristote aux neurosciences, Paris, Fayard, 2010, p. 295.

3. Nous, animaux et humains, Paris, Françoise Bourin, 2011, p. 8-9. 
cipent au même titre que nous au phénomène de la vie. Or, comme le suggèrent les analyses de l'auteur, l'enjeu de cet élargissement concerne peutêtre moins le statut éthique de la vie animale que la question du fondement du droit, dont il opère inévitablement une redéfinition. Accepter de représenter nos droits comme des droits du vivant plutôt que comme des droits humains, c'est se résoudre à choisir le plus petit dénominateur commun - à savoir la vie - pour penser le fondement du droit. La question est alors de savoir quelle politique pourra se fonder sur une redéfinition du domaine du droit qui a accepté de placer à son principe une vie dépourvue de tout contenu concret. Car ouvrir le droit aux animaux ne sera possible que dans la mesure où nous leur étendons nos droits, où nous les accueillons dans la sphère de notre moralité. En procédant ainsi, ne faisons-nous pas encore une fois preuve d'un anthropocentrisme indécrottable dont les éthiques animales ne parviennent pas plus que l'ancienne métaphysique à nous affranchir? Que nous choisissions ou non d'aligner le fondement du droit sur ce "plus petit dénominateur commun» qui nous rattache à l'ensemble des êtres vivants - quelle que soit la façon dont nous le concevrons - nous ne modifierons en rien le caractère inéluctablement humain de la politique, la nôtre comme celle des animaux: "La politique des animaux ne sera pas faite par les animaux mais par l'homme» (p. 125). Nier l'anthropocentrisme que cette indépassable évidence implique relèverait d'une forme de déni auquel l'auteur veut opposer l'élaboration d'une forme élargie d'anthropocentrisme, susceptible de s'accorder avec l'horizon d'une intégration réfléchie des animaux dans la communauté humaine ne remettant nullement en question le rôle fondamental que l'homme est appelé à y jouer.

\section{La légitimation philosophique}

La troisième et dernière ligne de force du zoocentrisme répond à une attente d'ordre philosophique - celle de voir définitivement rejetés et écartés les fondements métaphysiques de l'anthropocentrisme - , qui signe et ratifie l'orientation violemment antimétaphysique du paradigme zoocentriste. Nous avons déjà évoqué les raisons que l'on peut avoir de douter non pas du bien-fondé de ce type d'attente, expliquant le retour en force du naturalisme dans la prise en charge de la question anthropologique, mais de la capacité de ce programme naturaliste à renouveler en profondeur les termes à l'aide desquels s'articule cette question, là où il semble au mieux seulement susceptible de déplacer en les répétant à l'intérieur d'un nouveau type de discours (sous caution scientifique) les présupposés que générait le cadre d'élaboration métaphysique de ce questionnement. Sous couvert d'une critique radicale de la métaphysique héritée du renouvellement de la question anthropologique dont la révolution darwinienne a été porteuse, le zoocentrisme se donne toute licence pour déployer une description de l'homme qui renoue avec une forme non critique d'objectivisme offrant une représentation purement extérieure de nous-mêmes, comme si cette vie à laquelle 
l'homme se voit réduit n'était pas toujours en même temps vécue par lui en première personne. Nous découvrons alors, au fil de l'analyse que propose l'auteur de cette radicalisation antimétaphysique de la question anthropologique, le tableau apocalyptique d'une représentation de l'homme qui lui ou plutôt qui nous - échappe complètement: l'ère du zoocentrisme marque ainsi l'apogée d'une forme de pensée avec laquelle nous avons renoncé à être les acteurs de notre connaissance de nous-mêmes.

Cette dernière critique constitue la charnière de l'ouvrage de Bimbenet et permet de mettre en place une forme de renversement nous offrant l'occasion de tirer un parti positif des trois grandes critiques que nous venons de restituer. En mettant en valeur la dimension subjective de l'expérience humaine que le zoocentrisme se condamne à ignorer, la seconde partie de l'essai tente alors de répondre au défaut fondamental de cette forme de pensée fondamentalement "désengagée» au moyen de laquelle l'homme contemple sa propre vie de l'extérieur. L' "analytique resubjectivée de la vie humaine» que Bimbenet propose, et qu'il définit volontiers comme une autre option, faisant le choix d'élargir l'anthropocentrisme plutôt que de lui refuser toute forme de légitimité, doit ainsi avoir vocation à repenser notre relation à l'animalité à partir de notre savoir phénoménal de nous-mêmes et dans l'horizon de la constitution d'un monde commun dont ne seront plus exclus par principe les animaux. S'efforçant de construire une forme originale de «navigation" allant et venant de l'un à l'autre et obéissant à l'impératif de maintenir un regard lucide sur l'homme comme sur l'animal, l'ouvrage de Bimbenet représente une tentative pour ouvrir une nouvelle voie permettant de penser leur altérité et leur différence dans le geste même qui révèle leur proximité et engage le passage de l'un à l'autre. La mise en évidence de la complémentarité entre ces deux façons de penser la place de l'homme au sein du monde animal doit ainsi permettre d'établir un mode de relation aux animaux bâti sur une connaissance meilleure et plus lucide de notre origine animale - une origine à laquelle il est possible d'accorder un sens humain, fondé sur un "savoir phénoménal » de nous-mêmes.

Ce savoir est notamment rendu possible par notre pratique des formes de la socialité, à travers laquelle ne se déterminent pas seulement nos rapports à autrui — c'est-à-dire aux autres êtres humains avec lesquels nous partageons une existence sociale - dans la mesure où elle contribue aussi bien à nous permettre de définir les enjeux et la signification d'une vie proprement humaine. En inscrivant sa réflexion dans le prolongement des analyses phénoménologiques de l'intersubjectivité, Bimbenet insiste sur la spécificité des formes d'expérience de la vie sociale, et propose de penser la vie spécifiquement humaine comme une vie placée sous le signe de la constitution d'un monde commun. «La vie humaine, écrit-il, est une vie qui s'emporte au-delà d'elle-même en direction du monde commun» (p. 275). L'intérêt porté à l'expérience concrète des formes humaines de la vie sociale interdit selon lui de réduire l'analyse de la socialité aux modèles théoriques proposés tant par 
les primatologues que dans le cadre des théories de l'esprit issues de la psychologie cognitive, dans la mesure où il refuse de comprendre la société comme une simple «somme de corps atomisés, extérieurs les uns aux autres ", et de réduire le lien social au face à face abstrait d'individus que l'on devrait ainsi supposer originairement isolés et séparés les uns des autres. Au lieu de penser la socialité humaine à partir d'une forme de socialité animale minimale qui en constituerait à la fois l'origine et le modèle, il devient alors possible de comprendre ce monde commun, à la constitution duquel œuvrent dans leur ensemble les pratiques sociales des hommes, comme un monde ayant vocation à être élargi pour s'étendre, dans la mesure où nous avons des raisons de le faire, aux animaux, qui ne sauraient en être par principe exclus. Le rapport de l'homme à son animalité et aux autres animaux peut ainsi être refondé à partir de cette vie commune revendiquant une forme non métaphysique d'universalité, et qui constitue l'horizon de signification de l'existence humaine.

La force incontestable de la réflexion qu'Étienne Bimbenet s'efforce de systématiser dans cet ouvrage tient à sa façon précise et argumentée de louvoyer entre les inconséquences du zoocentrisme, qu'il permet de pointer et de repérer, pour y installer les bases d'une conception originale et subtile du rapport de l'homme à sa propre animalité. L'essentiel de l'argumentation mise en place par l'auteur tend ainsi à analyser aussi précisément que possible les implications philosophiques du zoocentrisme pour pouvoir y répondre philosophiquement, en construisant une articulation plus fine des relations entre humanité et animalité. Mais en s'attachant à apporter une réponse aux tensions qui sous-tendent les fondements théoriques du zoocentrisme, l'ouvrage de Bimbenet tend à délaisser une deuxième voie d'analyse qui affleure parfois à sa lecture et qui met en valeur un aspect fondamental de la question dont il s'occupe. L'un des diagnostics de fond sur lequel s'appuie la réflexion de Bimbenet est en effet que «le naturalisme nous en apprend davantage sur nous-mêmes, aujourd'hui, que sur la science en train de se faire» (p. 61), thèse capitale sur laquelle repose la possibilité de reprendre à nouveaux frais la question des rapports entre l'homme et l'animal en repartant non pas seulement des acquis scientifiques en la matière, mais aussi et surtout de la signification et des enjeux que cette question représente pour la compréhension que nous nous donnons de nous-mêmes. Ce qu'une telle analyse fait très justement apparaître, c'est que l'un des enjeux fondamentaux d'une investigation critique sur le zoocentrisme contemporain est d'abord de proposer une réflexion sur la forme d'aliénation très particulière que ce naturalisme enveloppe, dans la mesure où la position théorique derrière laquelle le zoocentrisme s'abrite ne peut éviter de tendre, le plus souvent de façon insidieuse, à redéfinir le sens et les limites de notre compréhension de nous-mêmes. Or, cette question, celle de savoir non seulement ce que le naturalisme peut nous apprendre de nous-mêmes, mais ce que son étude permet de mettre en évidence en révélant une figure peut-être inédite 
de l'auto-aliénation au moyen de laquelle l'homme accepte de redéfinir et de limiter la signification de son existence, ne trouve pas véritablement de réponse dans l'argumentation de Bimbenet. On aurait pu souhaiter que ses critiques se prolongent par une analyse des causes de cette renaturalisation problématique du savoir pointée avec justesse par l'auteur, en se demandant s'il ne faut pas lire le zoocentrisme comme un symptôme: le symptôme d'une forme nouvelle d'aliénation au moyen de laquelle l'homme cherche peut-être une nouvelle fois, pour reprendre les termes dans lesquels Feuerbach analysait le sens de la théologie chrétienne, à se cacher à lui-même sa propre essence.

L'ouvrage de Bimbenet ouvre toutefois des pistes pouvant permettre d'apporter à cette question certains éléments de réponse, en insistant notamment sur l'idée selon laquelle le naturalisme engage un "oubli du subjectif ». Le souci d'objectivité caractéristique de la posture naturaliste engage en effet, selon Bimbenet, un point de vue sur l'humain qui lui impose d'adopter une perspective extérieure sur lui-même, exclusivement en troisième personne, et qui signifie le rejet des méthodes d'analyse intégrant le point de vue en première personne au moyen desquelles les sciences humaines s'étaient notamment donné pour tâche de rendre compte du sens que l'homme donne à son expérience (p. 66). Le nouveau naturalisme qui définit la ligne idéologique dont procède le zoocentrisme peut ainsi être caractérisé par son incapacité à articuler les points de vue objectif, et subjectif, laquelle peut permettre d'en comprendre le succès manifeste tout autant que les limites intrinsèques. La profession de foi naturaliste du zoocentriste donne des gages de légitimité incontestables à son discours sur l'homme, dans la mesure où il est "objectivement vrai que nous soyons des animaux [...] en vertu des méthodes d'objectivation scientifique instruites par la biologie de l'évolution, les neurosciences cognitives ou l'éthologie et la primatologie». Mais en passant sous silence le versant subjectif de la vérité objective qu'il professe, et en oubliant d'articuler l'une à l'autre ces deux dimensions complémentaires de l'analyse, le zoocentrisme laisse aussi apparaître les limites problématiques de son discours, qui reste "subjectivement faux» dans la mesure où il s'interdit de rendre compte du "sens vécu de notre humanité (p. 70).

Cette analyse permet-elle pour autant de rendre compte des motifs qui confèrent à l'interprétation de l'homme véhiculée par le zoocentrisme l'autorité qui lui est aujourd'hui presque unanimement reconnue? Il est permis d'en douter, dans la mesure où l'ouvrage de Bimbenet analyse cet "oubli du subjectif» plutôt comme une conséquence problématique du naturalisme zoocentriste que comme le motif qui œuvre de façon plus ou moins discrète à sa légitimation. La réflexion critique fine et nuancée de Bimbenet suscite ainsi inévitablement une interrogation sur les raisons qui pourraient nous permettre de comprendre que nous puissions nous "projeter» dans une forme d'humanité décrite comme un composé de neurones, de gènes, et nous satisfaire d'une histoire biologique ramenant l'homme à sa parenté avec les 
grands singes. N'est-ce pas l'analyse de ces raisons qui pourrait délivrer, audelà des réserves que l'argumentation philosophique permet d'adresser aux fondements théoriques du zoocentrisme, une clé de compréhension de la forme peut-être inédite d'auto-aliénation dont le zoocentrisme est le symptôme: celle qui peut amener l'homme à se contempler béatement dans une parodie de lui-même et à s'identifier, pour des raisons qui restent à éclaircir, à une figure désarticulée, désincarnée, et fantomale de lui-même. Ce n'est pas le moindre mérite du livre d'Étienne Bimbenet que d'avoir donné ses gages de légitimité à cette question, au seuil de laquelle nous conduit sa réflexion. 